\title{
The 4-dimensional Langevin approach to low energy nuclear fission
}

\author{
F.A. Ivanyuk ${ }^{1,2, \star}$, C. Ishizuka ${ }^{2}$, M.D. Usang ${ }^{2}$, and S. Chiba ${ }^{2}$ \\ ${ }^{1}$ Institute for Nuclear Research, Kiev, Ukraine \\ ${ }^{2}$ Institute of Innovative Research, Tokyo Institute of Technology, Tokyo, Japan
}

\begin{abstract}
We applied the four-dimensional Langevin approach to the description of fission of ${ }^{235} \mathrm{U}$ by neutrons and calculated the dependence of the excitation energy of fission fragments on their mass number. For this we have fitted the compact just-before-scission configuration obtained by the Langevin calculations by the two separated fragments and calculated the intrinsic excitation and the deformation energy of each fragment accurately taking into account the shell and pairing effects and their dependence on the temperature and mass of the fragments. For the sharing of energy between the fission fragments we have used the simplest and most reliable assumption - the temperature of each fragment immediately after the neck rupture is the same as the temperature of mother nucleus just before scission. The calculated excitation energy of fission fragments clearly demonstrates the saw-tooth structure in the dependence on fragment mass number.
\end{abstract}

\section{Introduction}

One of the most successful approaches to the description of fusion-fission reactions and the fission process is the approach based on the Langevin equations for the shape degrees of freedom. In this approach instead of co-ordinates of individual nucleons one introduces the parametrization of the shape of nuclear surface and solves the dynamical equations for the time evolution of deformation parameters and conjugated momenta. The internal state of the system in such calculations is assumed not to deviate much from the thermal equilibrium. Up to now the shape of fissioning system was described mainly by three deformation parameters that specify the main fission degrees of freedom - the total elongation, the mass asymmetry and the neck radius of the fissioning system. In some works the fourth degree of freedom was introduced for the axial asymmetry or for the orientation of approaching ions. Meanwhile, it was pointed out a long ago by V. Pashkevich [1] that the deepest valley on the deformation energy surface of heavy nuclei corresponds to the configuration when one "fragment" is almost spherical, and another - very elongated. Such configuration corresponds to very low energy due to the large shell correction of almost spherical part (double magic ${ }^{132} \mathrm{Sn}$ ) and relatively small Coulomb interaction between spherical and elongated parts. Such configuration is impossible to describe within the three-parametric shape parametrization.

That is why recently [2] we took into account one more deformation parameter. Namely, we describe the shape of fissioning system by the two-center shell model (TCSM) parametrization in which

^e-mail: ivanyuk@kinr.kiev.ua 
the deformation parameters $\delta_{1}$ and $\delta_{2}$ are considered as independent dynamical variables. In addition, the mean field of Nilsson type was replaced by the Woods-Saxon potential that better reproduces the single-particle energies of heavy nuclei, especially at large deformations. Within this approach we have managed to reproduce rather accurately the mass and total kinetic energy distribution of fission fragments. We have also found a very clear correlation between the neutron multiplicity and the deformation of fission fragment just before scission.

The existing descriptions of excitation energy immediately after scission [3-6] are based on very different assumptions and approximations that even contradict to each other. So, the reason of the saw-tooth structure of the excitation energy and neutron multiplicity at present is not clear. In present work we use the results of the Langevin calculations in order to examine the dependence of fragments excitation energy on the its mass number and, thus, clarify the reason for the saw-tooth structure of neutron multiplicity.

\section{The Langevin approach}

We describe the fission process within four-dimensional Langevin approach [2, 7]. I.e., we solve the system of equations for time-dependent deformation parameters $q_{\mu}$ and conjugated momenta $p_{\mu}$,

$$
\frac{d q_{\mu}}{d t}=\left(m^{-1}\right)_{\mu \nu} p_{\nu}, \frac{d p_{\mu}}{d t}=-\frac{\partial V(q, T)}{\partial q_{\mu}}-\frac{1}{2} \frac{\partial}{\partial q_{\mu}}\left(m^{-1}\right)_{v \sigma} p_{\nu} p_{\sigma}-\gamma_{\mu \nu}\left(m^{-1}\right)_{\nu \sigma} p_{\sigma}+g_{\mu \nu} R_{\nu}(t),
$$

where the collective coordinates, $\left\{q_{\mu}\right\}=\left\{z_{0} / R_{0}, \delta_{1}, \delta_{2}, \alpha\right\}$ fix the shape of a fissioning nucleus within the two-center shell model shape parametrization [8]. Here $z_{0}$ is the elongation parameter of the nucleus, $R_{0}$ the radius of spherical compound nucleus, $\delta_{1}$ and $\delta_{2}$ are related to the deformation of fragments, and $\alpha$ is the mass asymmetry. The neck parameter $\epsilon$ is kept constant, $\epsilon=0.35$. The detailed description of the two-center shell model shape parametrization can be found in $[9,10]$.

For the tensors of mass and friction in four-dimensional calculations we used the macroscopic quantities - Werner-Wheeler method [11] for the mass tensor $m_{\mu v}$ and reduced $\left(k_{s}=0.27\right)$ wall-andwindow formula $[12,13]$ for the friction tensor $\gamma_{\mu \nu}$. The Langevin random force $g_{\mu \nu} R_{v}(t)$ is the product of white noise $R_{\nu}(t)$ and the temperature dependent strength factors $g_{\mu \nu}$. The factors $g_{\mu \nu}$ are related to the temperature and friction tensor via the modified Einstein relation [14],

$$
\sum_{\sigma} g_{\mu \sigma} g_{\sigma v}=T^{*} \gamma_{\mu v}, \quad \text { with } T^{*}=\frac{\hbar \varpi}{2} \operatorname{coth} \frac{\hbar \varpi}{2 T}, \quad \text { and } \hbar \varpi=2 \mathrm{MeV} .
$$

The temperature $T$ in this context is related to total excitation energy $E_{x}^{(t o t)}$ and intrinsic energy $E_{\text {int }}$,

$$
E_{\text {int }}=E_{x}^{(t o t)}-\frac{1}{2}\left(m^{-1}\right)_{\mu \nu} p_{\mu} p_{v}-V(q, T=0)=a T^{2},
$$

where $a$ is the level density parameter.

The potential, $V(q, T)$ used here was calculated with the deformed Woods-Saxon potential by the code of Pashkevich [1]. The shell corrections $\delta E_{\text {shell }}$ and $\delta E_{\text {pair }}$ were calculated for $T=0$. For the temperature dependence of the shell and pairing effects we adopt the approximation suggested in [15].

The Langevin calculations start at either the potential minimum (the ground state shape) or at the second minimum. As long as the results presented in this work are concerned, both choices gave identical results. Initially, the momentum $p_{\mu}$ 's were set to be zero, and Langevin motions are initiated by the conservative and random forces. Such calculations are continued until the trajectories reach the scission points, which are defined as points where the neck radius becomes zero. Calculations were repeated typically from $10^{5}$ to $10^{6}$ times, the final phase-space data were stored on a disk, and various quantities were calculated after all the Langevin calculations were finished. 


\section{The deformation and the intrinsic excitation energy}

One of the quantities of interest for this work is the average value of the deformation parameter $\delta_{1}$ (or $\delta_{2}$ ). An example of dependence of the average value of fragment deformation $\langle\delta\rangle$ on the mass asymmetry is shown in left part of Fig. 1. Please, note that within the TCSM shape parametrization the parameters $\delta_{1}$ and $\delta_{2}$ fix the deformation of the outer parts of "fragments" only, from the tips of nucleus to the thickest place. The deformation of the inner part, from the thickest place till the neck, is regulated by all 5 deformation parameters. At the scission point the nucleus is very elongated and the inner part is elongated too. In order to make the fragment as a whole more spherical, the outer part should be somewhat oblate, see the right part of Fig. 1. Thus, negative $\delta$ within TCSM shape parametrization does not mean that the whole fragment is oblate.

For the comparison we show also in the left part of Fig. 1 the neutron multiplicities $\bar{v}$ from works $[16,17]$. The average value $\langle\delta\rangle$ was shifted up by 0.25 in order to see better the similarity between $\bar{v}$ and $\langle\delta\rangle$. Indeed, one can see a surprising correlation between $\langle\delta\rangle$ and $\bar{v}$. Such similarity was noticed already in [18]. However, the similarity between the fragment deformation and $\bar{v}$ does not explain much. It is the fragment excitation energy that should be compared with $\bar{v}$, not the fragment's deformation. In order to calculate the fragment excitation energy the information on its shape is not sufficient. One should know also the sharing of excitation energy between fission fragments.

To clarify this question we calculate in present work the excitation energy of each fragment separately. This is not so trivial since from the Langevin calculations we can get only the excitation energy of the whole nucleus just before the scission. Very often it is assumed that the excitation energy is shared between fragments proportionally (or inversely proportionally) to their masses. Here we will not use none of such assumption but will calculate the excitation energy of each fragment directly, assuming that the temperature of both fission fragments immediately after scission is exactly the same as the temperature of mother nucleus just before scission.

In order to do this we fit the compact shape (with zero or finite neck) just before scission by two separate fragments. An example of such fit is shown in the right part of Fig. 1. As one can see from the figure, the fit can be done quite accurately.
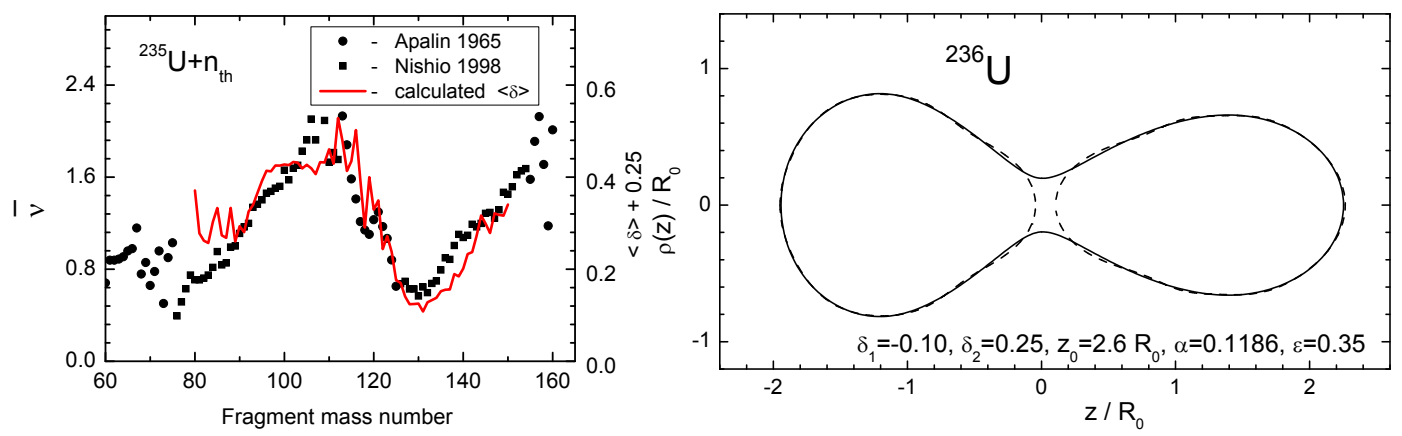

Figure 1. Left: the average value $\langle\delta\rangle$ of the fragment deformation (right scale) and the average neutron multiplicities $\bar{v}[16,17]$ as function of mass asymmetry (fragment mass number). Right: an example of the shape of fissioning system just before scission and its fit by the two separate fragments.

To check the accuracy of the fit we compare the liquid drop energies and the shell corrections calculated for the compact shape just before scission and for the fit by two separate fragments. As one can see from Fig. 2, both the liquid drop energy and the shell corrections calculated for the compact 
shape and the two separated fragments are very close to each another. This means that the replacement of the shape just before scission by separate fragments is quite accurate.

In case of compact shape the number of particles to the right or the left from the neck $(z=0)$ is not necessarily an integer number. After the fit the number of particles in each fragment must be integer. Thus, the $Z / N$ ratio in the compact shape and fragments is slightly different. This explains the wiggles of red curve in left part of Fig. 2. Due to the same reason the fragments may not be even-even nuclei (the mother nucleus ${ }^{236} \mathrm{U}$ is even-even) and the odd-even effects are seen in the shell corrections of fitted fragments.
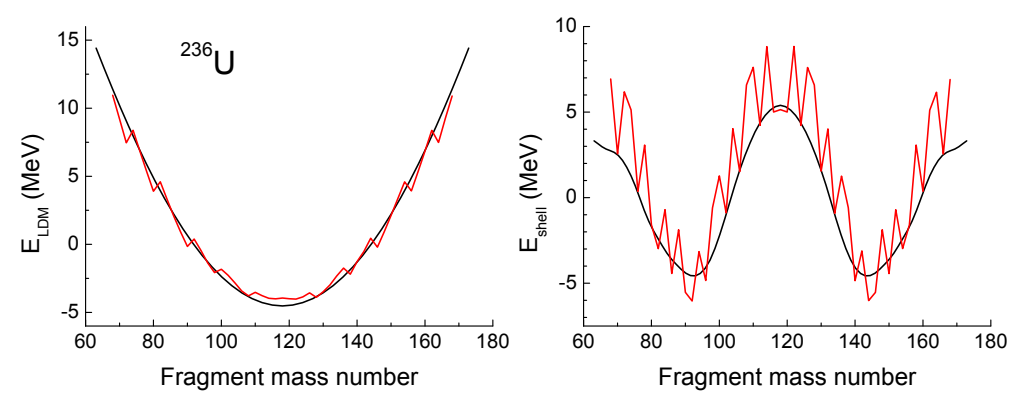

Figure 2. The liquid drop energy (left) and the shell correction at $T=0$ (right) calculated for the compact shape just before scission (black lines) and the fit by two separate fragments (red) as function of mass asymmetry (fragment mass number).

To calculate the excitation energy, especially the shell correction, at finite but small temperature one should be very cautious. The popular relations like $E_{x}=a T^{2}$ or $E_{\text {shell }}(T)=E_{\text {shell }}(T=0) \cdot \Phi(T)$ works somehow "on average" at large enough temperatures. At small temperature, say below $T=$ $1 \mathrm{MeV}$ both relations are not reliable. Their accuracy is hard to estimate.

In order to avoid the inaccuracy caused by the above relations we will not use them but will calculate the energy at finite temperature directly. I.e. we define the energy at finite temperature as the sum of the energy within independent particle model $E_{i p m}(T)$ and the pairing energy $E_{\text {pair }}$ defined within BCS approximation,

$$
E(T)=E_{i p m}(T)+E_{\text {pair }}(T),
$$

with

$$
E_{i p m}(T)=2 \sum_{k} \epsilon_{k} n_{k}(\mu, T), \text { with } n_{k}(, \mu, T)=\frac{1}{1+e^{\left(\epsilon_{k}-\mu\right) / T}}, \text { and } N=2 \sum_{k} n_{k}(\mu, T),
$$

and

$$
E_{\text {pair }}(T)=2 \sum_{k=N_{1}}^{N_{2}} \epsilon_{k}\left[n_{k}(\Delta, \lambda, T)-n_{k}(\mu, T)\right]-\Delta^{2} / G
$$

with

$$
n_{k}(\Delta, \lambda, T)=\frac{1}{2}\left(1-\frac{\epsilon_{k}-\lambda}{E_{k}} \tanh \frac{E_{k}}{2 T}\right), \sum_{k=N_{1}}^{N_{2}} n_{k}(\Delta, \lambda, T)=\sum_{k=N_{1}}^{N_{2}} n_{k}(\mu, T), \sum_{k=N_{1}}^{N_{2}} \frac{1}{E_{k}}=\frac{2}{G} .
$$

In (6)-(7) $G$ is the strength of pairing interaction, $N_{1}$ and $N_{2}$ are the limits of the pairing window and $E_{k}$ is the quasiparticle energy $E_{k}=\sqrt{\left(\epsilon_{k}-\lambda\right)^{2}+\Delta^{2}}$. 
For convenience one may add and subtract from (4) the Strutinsky averaged quantities $\widetilde{E}_{i p m}(T=0)$ and $\widetilde{E}_{p a i r}(T=0)$ and eventually apply the Strutinsky renormalization, i.e., replace the $\widetilde{E}_{i p m}(T=$ $0)+\widetilde{E}_{\text {pair }}(T=0)$ by the liquid drop energy $E_{L D M}$. Then the energy (4) attains the form

$$
E(T)=E_{L D M}+\left[E_{i p m}(T)-\widetilde{E}_{i p m}(T=0)\right]+\left[E_{p a i r}(T)-\widetilde{E}_{p a i r}(T=0)\right] .
$$

The intrinsic excitation energy $E_{x}(T)$ is defined in a usual way

$$
E_{x}(T)=E(T)-E(T=0) .
$$

One can see that the liquid drop energy and average $\widetilde{E}_{i p m}(T=0)$ and $\widetilde{E}_{\text {pair }}(T=0)$ do not contribute to the excitation energy. That is only the temperature dependent $E_{i p m}(T)$ and $E_{p a i r}(T)$ that matter. These quantities are completely defined by Eqs. (5)-(7) and can be calculated for any temperature.

The dependence of the excitation energy on the temperature and the mass number is shown in Fig. 3. One can see that dependence of $E_{x}$ on temperature $T$ differs substantially from the popular approximation $E_{x}=a T^{2}$. Even more important is the dependence of $E_{x}$ on the mass number at fixed temperature. Only at very high temperature, above $T=3 \mathrm{MeV}$, the excitation energy is proportional to the mass of nucleus. Below $T=1 \mathrm{MeV}$ the dependence is more complicated. Say, at $T=0.5 \mathrm{MeV}$ the excitation energy is almost zero around double magic ${ }^{132} \mathrm{Sn}$ and much larger away from $A=132$, both at larger and smaller $A$. Thus, the fragment with mass number smaller than $A=132$ can have larger intrinsic excitation energy (compared with $A=132$ ) even if the temperature of both is the same. This circumstance has an important effect on the mass dependence of the excitation energy, see below.
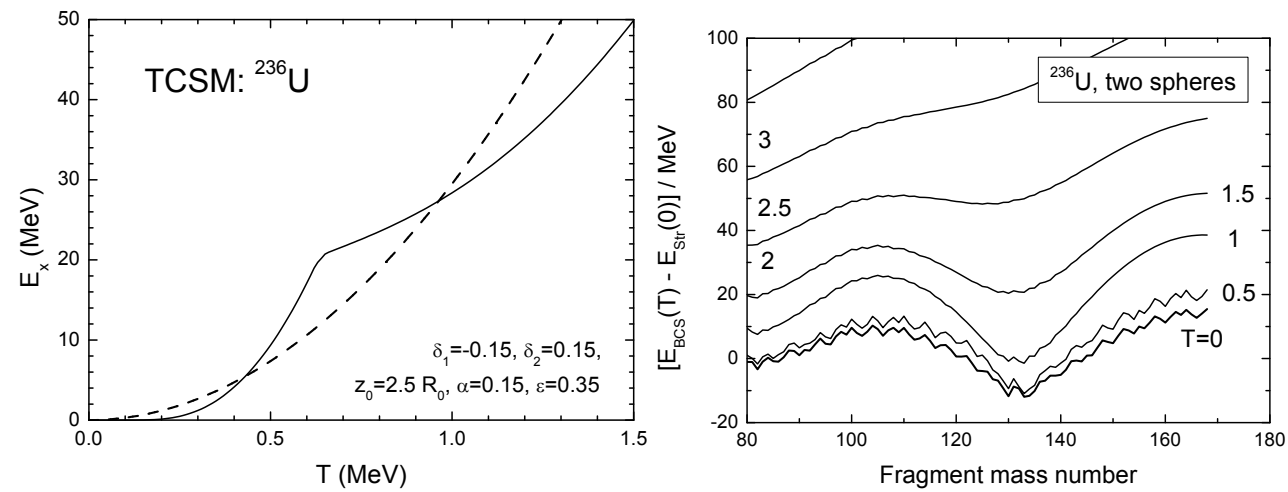

Figure 3. Left: the excitation energy (9) versus temperature for the scission shape given by $z_{0}=2.5 R_{0}, \delta_{1}=$ $-0.15, \delta_{2}=0.15, \alpha=0.15, \epsilon=0.35$. The dash curve - the approximation for the excitation energy $E_{x}=a T^{2}$, with $a=A / 8$. Right: the dependence of energy (8) (minus $E_{L D M}$ ) on the mass of spherical fragment. The $Z / N$ ratio of the fragment is chosen the same as for ${ }^{236} \mathrm{U}$.

\section{The total kinetic energy and the neutron multiplicity}

Having at our disposal the results shown in Fig. 3 we are ready to calculate the excitation energy of fission fragments as a function of their mass number. Because of random force in Langevin equations the calculations are repeated many times. Some trajectories reach the scission line, some not. Each 
trajectory reaching the scission line corresponds to a certain shape and temperature. These results are shown by the points in the left-upper part of Fig. 4. The solid line represent the average value of temperature at fixed mass asymmetry.

For each point in the left-upper part of Fig. 4 (the shape and temperature) we fit the pre-scission shape by two separate fragments, like it is shown in Fig. 1, and calculate the excitation energy (9) of each fragment for the temperature given by the output of Langevin calculations. The average value of intrinsic excitation energy (9) (red) is shown in right-upper part of Fig. 4 as function of mass asymmetry. It may seems unexpected, but this curve demonstrates the saw-tooth structure without assuming that the light fragment is hotter. This feature can be understood looking at Figs. 1, 3. The value of excitation energy at given temperature depends very much on the density of states around Fermi energy. Around $A=132$ the shape of heavy fragment is close to spherical, see right part of Fig. 1. The density of states around Fermi energy is very low (because ${ }^{132} \mathrm{Sn}$ is a double magic nucleus). Thus, the excitation energy around $A=132$ is the smallest. The fragment with $A \approx 120$ is a middle-shell nucleus, and it is very deformed. The density of states around Fermi energy is high in this case and the excitation energy is large. This explains the sudden drop of the excitation energy from $A \approx 120$ to $A \approx 132$ at constant temperature. Besides, the average temperature for symmetric fission is slightly larger compared with asymmetric, see left-upper part of Fig. 4.
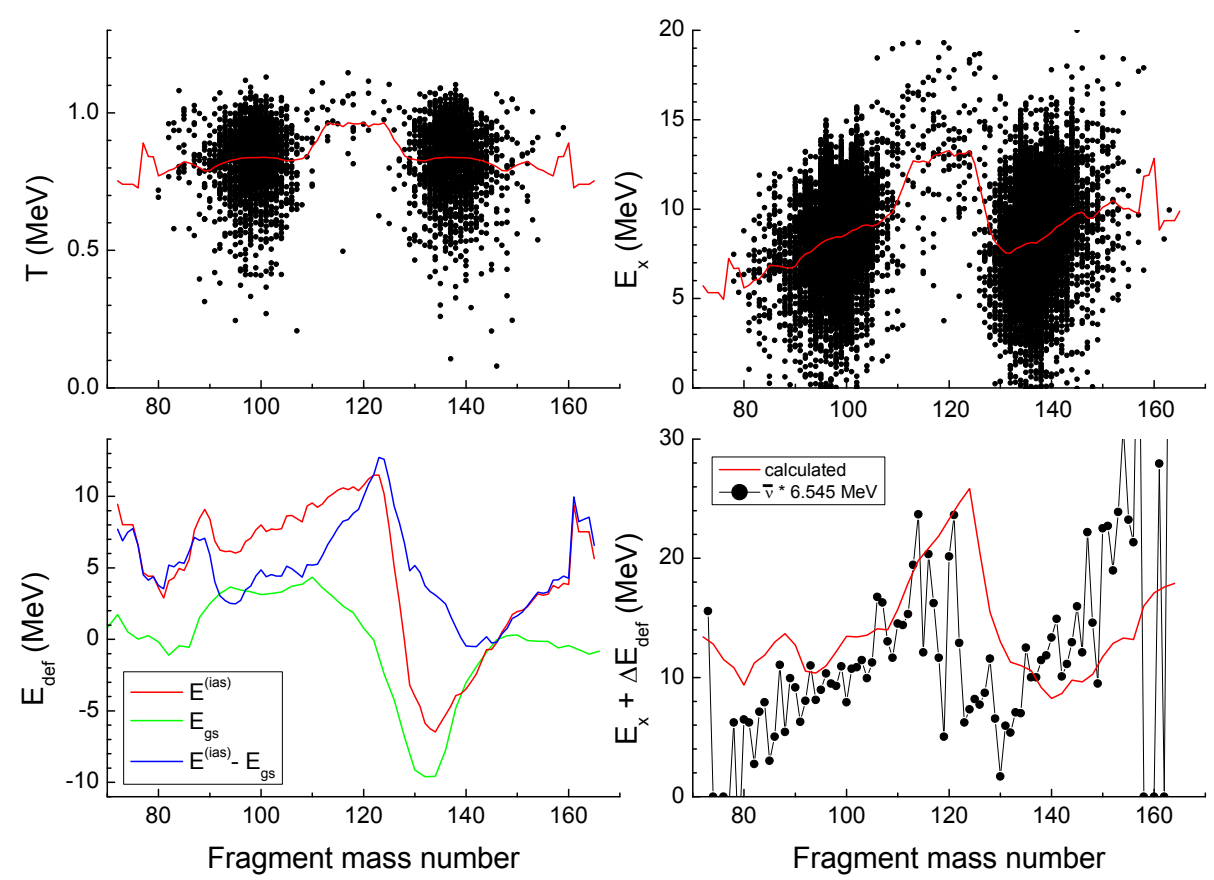

Figure 4. Upper left: distribution of temperature just before scission of ${ }^{236} \mathrm{U}$ for $E_{n}=5 \mathrm{MeV}$. The solid curve is the average temperature for given mass asymmetry. Upper right: the intrinsic excitation energy (9). Bottom left: the deformation energy immediately after scission $E^{(i a s)}$ (red), the ground state deformation energy $E_{g s}$ (green) and the extra deformation energy $\Delta E_{d e f}=E^{(i a s)}-E_{g s}$ (blue). Bottom right: the total excitation energy $E_{x}+\Delta E_{d e f}$ (red) and the neutron multiplicity [19] multiplied by the neutron separation energy $S_{n}=6.545 \mathrm{MeV}$. 
Another contribution to the excitation energy comes from the extra deformation energy $\Delta E_{\text {def }}=$ $E^{(i a s)}-E_{g s}$, i.e. the difference between the energy $E^{(i a s)}$ immediately after scission configuration (at $T=0)$ and that in the ground state $E_{g s}$. The fragment deformation energy $E^{(i a s)}$ is shown in the leftbottom part of Fig. 4. Similar to the intrinsic excitation energy it exhibits the saw-tooth behaviour. Around $A \approx 132$ it is small (because the fragment is almost spherical) and away from $A \approx 132$ it is large (because the fragment is very deformed). The problem with the deformation energy is that the ground state energy is also minimal at $A=132$. So, the extra deformation energy $\Delta E_{\text {def }}$ does not show much of saw-tooth structure.

Still, the sum of intrinsic excitation energy and extra deformation energy $E_{x}+\Delta E_{d e f}$ clearly shows the saw-tooth behaviour very similar to experimental results for $\bar{v}$, see the right-bottom part of Fig. 4 .

\section{Summary}

The saw-tooth structure in the fragments excitation energy is a natural consequence of shell effects in the deformation and intrinsic excitation energies at low excitations (temperatures). This conclusion is based on the results of four-dimensional Langevin calculations that lead to the realistic configuration at the scission point - the heavy fragment is almost spherical and light - very deformed. The only assumption is: just before scission the temperature is constant over the whole volume of a fissioning nucleus; immediately after neck rupture each fragment has the same temperature as it was just before scission. The calculated sum of the two contributions to the total excitation energy (deformation and excitation at scission) for ${ }^{236} \mathrm{U}$ as a function of the fragment mass clearly shows a saw-tooth behaviour in qualitative agreement with the measured dependence of the prompt neutron multiplicity.

This contribution includes the results of "Comprehensive study of delayed-neutron yields for accurate evaluation of kinetics of high-burn up reactors" entrusted to the Tokyo Institute of Technology by the Ministry of Education, Culture, Sports, Science and Technology of Japan (EXT). We appreciate very much the informative discussions with Dr. C. Schmitt and Prof. A. Tudora.

\section{References}

[1] V.V. Pashkevich, Nucl. Phys. A 169, 275 (1971)

[2] C. Ishizuka, M.D. Usang, F.A. Ivanyuk, J.A. Maruhn, K. Nishio, S. Chiba (submitted to PRC)

[3] K.-H. Schmidt and B. Jurado, Phys. Rev. Lett. 104, 212501 (2010)

[4] K.-H. Schmidt and B. Jurado, Phys. Rev. C 83, 061601(R) (2011)

[5] C. Morariu, A. Tudora, F.-J. Hambsch et al., J. Phys. G. Nucl. Part. Phys. 39, 055103 (2012)

[6] A. Tudora, F.-J. Hambsch, I. Visana, G. Giubega, Nucl. Phys. A 940, 242 (2015)

[7] Y. Abe, S. Ayik, P.-G. Reinhard and E. Suraud, Phys. Rep. 275, 49 (1996)

[8] J. Maruhn and W. Greiner, Z. Phys. 251, 431 (1972)

[9] F.A. Ivanyuk, S. Chiba, and Y. Aritomo, Phys. Rev. C 90, 054607 (2014)

[10] M.D. Usang, F.A. Ivanyuk, C. Ishisuka, and S. Chiba, Phys. Rev. C 94, 044602 (2016)

[11] K.T.R. Davies, A.J. Sierk, J.R. Nix, Phys. Rev. C 13, 2385 (1976)

[12] J. Blocki, Y. Boneh, J.R. Nix et al., Ann. Phys. 113, 330 (1978)

[13] A.J. Sierk and J.R. Nix, Phys. Rev. C 21, 982 (1980)

[14] H. Hofmann, D. Kiderlen, Int. Jour. Mod. Phys. E 7, 243 (1998)

[15] J. Randrup and P. Moller, Phys. Rev. C 88, 064606 (2013)

[16] V.F. Apalin, Yu.N. Gritsyuk, I.E. Kutikov et al., Nucl. Phys. 71, 553 (1965) 
[17] K. Nishio, Y. Nakagome, H. Yamamoto, I. Kimura, Nucl. Phys. A 632, 540 (1998)

[18] B.D. Wilkins, E.P. Steinberg, and R.R. Chasman, Phys. Rev. C 14, 1832 (1976)

[19] R. Müller, A.A. Naqvi, F. Käppeler, and F. Dickmann, Phys. Rev. C 29, 885 (1984) 